\title{
Evaporatively-driven Marangoni instabilities of volatile liquid films spreading on thermally conductive substrates ${ }^{\text {is }}$
}

\author{
Pirouz Kavehpour ${ }^{\mathrm{a}, *}$, Ben Ovryn ${ }^{\mathrm{b}}$, Gareth H. McKinley ${ }^{\mathrm{a}}$ \\ a Department of Mechanical Engineering, Massachusetts Institute of Technology, Cambridge, MA, USA \\ ${ }^{\mathrm{b}}$ Department of Biomedical Engineering, Case Western Reserve University, Cleveland, OH, USA
}

\begin{abstract}
Laser confocal microscopy is used to non-invasively investigate the steady and unsteady evolution of viscous microdroplets on solid substrates. Three characteristic dynamical regimes of spreading drops (viscous-capillary, viscous-inertia-capillary, and inertia-capillary) are studied using this non-invasive optical technique. It is shown that the dynamics of each regime depend on the Ohnesorge number, $\mathrm{Oh}=\mu /(\rho R \sigma)^{\frac{1}{2}}$, and on the relative magnitudes of the droplet height, radius, compared with the capillary length, $\ell_{\text {cap }}=\sqrt{\sigma / \rho g}$. The power-law relationships between the extent of spreading and elapsed time that are extracted from the experiments are in excellent agreement with available analytical results. We also study the onset and evolution of surface instabilities of the slightly volatile liquid films as they spread across the thermally-conductive surfaces. When the fluid droplet is a volatile silicone oil and the surface is a smooth silicon wafer, an evaporatively-driven thermocapillary instability leads to onset of a time-dependent free surface motion. Below a certain critical thickness $(\sim 20 \mu \mathrm{m})$, waves can be observed on the free surface of the film, and the confocal technique is used to measure the amplitude, the frequency, and non-linear evolution of these waves. We interpret these waves in terms of evaporatively-driven Marangoni instabilities induced by surface tension gradients close to the moving contact line. Experiments show that the amplitude and the critical onset thickness of the disturbances vary with the viscosity and the volatility of the liquid, and also with the surface roughness and thermal diffusivity of the substrate. The critical onset conditions for this evaporatively driven instability can be characterized by a dimensionless interfacial thermal resistance, $\mathfrak{R}$, which has to be larger than a critical value at the onset of instability. We also demonstrate that this evaporatively-driven Marangoni instability can be eliminated by reducing the volatility of the liquid or the thermal diffusivity of the substrate. (C) 2002 Elsevier Science B.V. All rights reserved.
\end{abstract}

Keywords: Spreading viscous drop; Marangoni instability; Optical techniques; Volatile films; Evaporation

\footnotetext{
Submitted for publication in the proceedings of The International TRI/Princeton Workshop on "Nanocapillarity: Wetting of Heterogeneous Surfaces and Porous Solids" as a special issue of Colloids and Surfaces A: Physicochemical and Engineering Aspects.

* Corresponding author.
}

\section{Introduction}

Understanding the dynamics of liquids spreading on solids is of importance in a wide variety of industrial applications such as coating processes, soldering technology, and the printing of inks. Studies of the motion of a spreading axisymmetric drop on a smooth horizontal substrate provide a 
good benchmark model for many of these types of problems. The final equilibrium configuration of the drop may be a thin uniform film covering the substrate ('perfect wetting') or a drop of finite lateral extent ('partial wetting') depending on the relative magnitudes of the interfacial tensions between the solid, liquid and vapor acting at the three phase contact line (Adamson and Gast [1]).

In addition to studies of the final static shape and equilibrium energetic configuration, considerable interest exists in understanding the dynamical process of the spreading of the liquid droplets because, in the majority of commercial processes, a wetting or partially-wetting droplet is rarely deposited in its final equilibrium shape. There are several important parameters that govern the dynamical process of spreading and the final shape of the drop. These include the initial drop volume, $\Omega$, the shear viscosity, $\mu$, surface tension, $\sigma$, and the density, $\rho$, of the liquid plus the surface roughness of substrate, $\varepsilon$. Other parameters such as the volatility of the liquid and the temperature difference between the substrate and the droplet can also be very important since the thermophysical properties of liquids can be a strong function of temperature.

A fluid droplet deposited on a substrate rapidly forms a thin film with a lateral extent $R(t)$ greatly exceeding its characteristic thickness $h(t)$ and consequently lubrication analysis can often be used to understand the dynamics of the spreading process. Experiments and analysis show that the lateral size of the spreading droplet and the steady rate of spreading are frequently a power-law function of elapsed time (Brochard and de Gennes [2]). A common example is 'Tanner's law' (Tanner [3] and de Gennes [4]) which relates the lateral rate of spreading $(\dot{R}(t))$ to the dynamic contact angle of the interface $\left(\theta_{\mathrm{a}} \sim h(t) / R(t)\right)$. The power-law exponent characterizing this spreading is a sensitive function of the dominant physical forces (e.g. capillary, viscous, inertia, and gravitational) that are driving and resisting the spreading. A detailed review of these regimes is provided by Oron et al. [5]. These results can also be summarized quite succinctly in terms of a spreading diagram such as that shown in Fig. 3 which we discuss in detail below.
Inertial effects in a spreading film can be neglected if the dimensionless Ohnesorge number $\mathrm{Oh}=\mu / \sqrt{\rho R \sigma}$ is much greater than one. The spreading regime then depends on the relative lateral extent, $R$, and characteristic height of the drop, $h$, compared with the intrinsic capillary length of the system, $\ell_{\text {cap }}=\sqrt{\sigma / \rho g}$, over which gravitational effects are important. Huppert [6] and Cazabat and Stuart [7] have examined these regimes extensively. When inertial effects are important, different power law and a different spreading diagram is obtained. This situation was considered quite early in the context of oil films spreading rapidly on the ocean (see Fay [8] and Hoult [9] for additional details).

Other experiments have been performed to investigate the effects of the substrate on the motion of the three-phase contact line and the rate of spreading. For example, Ehrhard [10] investigated the dynamical changes induced by a non-isothermal substrate and Cazabat and Cohen Stuart [7] demonstrated the effect of surface roughness on the power-law spreading of liquid drops.

The studies discussed above have all assumed that the spreading profile of the liquid drop is stable in time and remains axisymmetric as spreading proceeds. However, it is also frequently observed that the spreading film may become unstable and develop a spatially-periodic fingering pattern or 'festoon instability' (see Redon et al. [11] and Fanton and Cazabat [12]). Elimination or control of such hydrodynamic instabilities is important in painting, coating and inkjet printing.

These interfacial instabilities are frequently driven by local variations in the surface tension, $\sigma$, which controls the rate of spreading. Such variations can arise naturally due to the dependence of the surface tension on the temperature field and concentration of dissolved solutes, and the resulting phenomena are collectively referred as Marangoni effects (Scriven and Sternling [13]). When unsteady flows arise as a result of thermal variations perpendicular to the fluid interface, the resulting unsteady flows are usually called Marangoni instabilities. If instability occurs because of an applied temperature field parallel to the interface of the liquid film, the motion is typically referred to as hydrothermal instability 
(see for example the studies of Smith and Davis [14] and [15]). Detailed reviews of both of these classes of instabilities can be found in Davis [16] and Schatz and Neitzel [17].

A separate class of Marangoni flows arises from evaporation. Thermal energy is removed from the liquid resulting in local changes in the temperature and thus also in surface tension. Since the more volatile components of a mixture evaporate most rapidly, concentration gradients also develop. Furthermore, since the evaporative flux is largest near a contact line, spatial gradients in the concentration and/or temperature can drive strong secondary flows. Such evaporatively-driven Marangoni flows lead to formation of coffee rings (Deegan et al. [18]) and also wine tears and tear ducts (Hosoi and Bush [19]). These flows also have been proposed as a way to elongate DNA chains for subsequent sequencing analysis $(\mathrm{Hu}$ and Larson [20]) and to enhance heat transfer from menisci in inclined capillary tubes (Mirzamoghadam and Catton [21]).

Such instabilities have been studied in the past in static geometries for which the base flow is stationary and both experimental and theoretical analysis is more tractable. However, evaporatively-driven Marangoni instabilities may also spontaneously arise in spreading droplets, especially if the drops are small so that the surface area/volume ratio is large, or if the droplets are polymeric in composition and have poor thermal conductivities. In order to successfully investigate such situations it is necessary to develop non-invasive techniques for rapidly and accurately probing the surface profiles of fluid microdroplets. Optical techniques are obviously well suited for such tasks. Fondecave et al. [22] attempted to use ellipsometry to spatially resolve the shape and thickness of spreading liquid films but reported major errors, which they speculated arose from surface heating by the laser and which drove Marangoni instabilities. A principal goal of this research is to investigate the physics behind such instabilities and determine the important parameters that affect this phenomenon.

In the first part of this paper we describe the optical measurement system and use it to investigate the steady spreading of fluid microdroplets in different regimes. The efficacy of the technique is tested by comparing our experimental results with the scaling predictions that are available in the literature. In the second part of the paper, we investigate the evaporative-Marangoni instabilities that develop in the liquid films when the spreading liquid is volatile and flows over a thermally-conductive solid substrate. The spatial and temporal characteristics of this instability depend very sensitively on the properties of both the substrate and the spreading fluid.

\section{Methods and materials}

\subsection{Fluids and substrates}

The three test fluids used in this study-denoted, for convenience, by the labels L (low viscosity), M (medium viscosity), H (high viscosity) - are constant-viscosity silicone oils with relatively low molecular weights which are produced by Gelest Inc. There are several advantages in using these silicone oils: first, they all wet the substrates that are used in our experiments; secondly, the viscosities of these liquids are approximately equal to the viscosity of water (L), ten times that of water (M) and one hundred times that of water $(\mathrm{H})$. This enables us to explore several regimes of spreading on viscous liquids on the surface. The general thermophysical properties of these liquids are given in Table 1. A few additional tests are performed with a fourth fluid denoted $V$ with a viscosity of $10 \mathrm{~Pa}$ s. Rheological measurements show that all four test fluids have a constant viscosity for deformation rates less than $300 \mathrm{~s}^{-1}$.

To investigate thermocapillary effects on the spreading of fluid microdroplets, one needs to quantify the effects of temperature variations on the thermophysical properties of the fluids, especially the surface tension $\sigma(T)$ and the shear viscosity $\mu(T)$. A Wilhelmy plate tensiometer (Krüss K10) is used to measure the surface tension of silicone oils as a function of temperature. Fig. 1(a) shows the effect of temperature on the surface tension of fluids L, M, and H. All fluids exhibit a linear decrease in interfacial tension with 
temperature of magnitude $\mathrm{d} \sigma / \mathrm{d} T \approx 10^{-4} \mathrm{~N} \mathrm{~m}^{-1}$ $\mathrm{K}^{-1}$. For $\mathrm{L}, \mathrm{M}$ and $\mathrm{H}$, the experiments show the following relationships: $\sigma_{\mathrm{L}}=0.016-7.63 \times 10^{-5}$ $\left(T-T_{0}\right), \quad \sigma_{\mathrm{M}}=0.018-8.28 \times 10^{-5} \quad\left(T-T_{0}\right), \quad$ and $\sigma_{\mathrm{H}}=0.021-9.5 \times 10^{-5}\left(T-T_{0}\right)$. Here, the reference temperature is taken as $T_{0}=300 \mathrm{~K}$.

The thermal variation in the steady shear viscosity of the test fluids was measured with a cone and plate rheometer (TA Instruments AR$1000 \mathrm{~N})$. The data for fluids $\mathrm{L}, \mathrm{M}$, and $\mathrm{H}$ as a function of temperature is presented in Fig. 1(b), and can be well described by an Arrhenius expression of the form $\mu(T)=\mu\left(T_{0}\right) \exp \left[\Delta H_{\mu} / \bar{R}\left(T^{-1}-\right.\right.$ $\left.\left.T_{0}^{-1}\right)\right]$. Values of the reference viscosity $\mu\left(T_{0}\right)$ and activation energy $\Delta H_{\mu} / \bar{R}$ for fluids L, M, and $\mathrm{H}$ are given in Table 1.

The composition and characteristics of the underlying substrate can also have major effects on the spreading process. The roughness, $\varepsilon$, and thermal diffusivity, $\alpha_{\mathrm{s}}$, are among the important properties of the solid substrate that can affect spreading and wetting. To explore these effects, the spreading dynamics on silicon, brass, copper (with rough and smooth surfaces), and glass substrates are investigated in the present study. The thermophysical properties of these surfaces are given in Table 2.

\subsection{Apparatus and experiment procedure}

Confocal microscopy is a very common technique for high-resolution optical measurements and is utilized in many applications from non-invasive study of biological tissues to surface roughness measurements (Corle and Kino [23]). Confocal surface metrology is based on a dynamic focus-detection technique together with a closedloop feedback system. The confocal surface metrology system (Keyence, LT-8110) differs from a conventional optical or confocal microscope in that it illuminates and images the sample one point at a time through a pinhole. A laser beam passing through a semi-silvered mirror is focused by the high numerical aperture objective lens to a diffraction-limited spot at the focal plane. If the light spot is focused on a surface by adjusting the objective lens vertically during the scanning process, then the displacement of the objective lens determines the vertical dimension of the surface topography. In the present system, the objective lens is dynamically refocused by a tuning fork in order to automatically maintain an optimal focus of the laser spot on the surface. Whenever the surface is in focus, the intensity of

Table 1

Thermal and physical properties of silicone oils used in the experiments

\begin{tabular}{|c|c|c|c|c|c|}
\hline & DMS-T01 & DMS-T11 & DMS-T21 & DMS-T41 & DMS-T07R \\
\hline a.k.a & $\mathrm{L}$ & M & $\mathrm{H}$ & $\mathrm{V}$ & NV \\
\hline$\mu_{\mathrm{f}}(\mathrm{Pa} \mathrm{s})$ & 0.001 & 0.01 & 0.1 & 10 & 0.007 \\
\hline$\rho_{\mathrm{f}}\left(\mathrm{kg} \mathrm{m}^{-3}\right)$ & 818 & 935 & 966 & 977 & 920 \\
\hline$\sigma_{\mathrm{f}}\left(\mathrm{N} \mathrm{m}^{-1}\right)$ & 0.0174 & 0.0201 & 0.209 & 0.210 & - \\
\hline 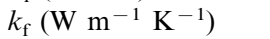 & 0.1256 & 0.1256 & 0.1256 & 0.1256 & 0.1256 \\
\hline$\alpha_{\mathrm{f}}\left(\mathrm{m}^{2} \mathrm{~s}^{-1}\right)$ & $10^{-7}$ & $9.2 \times 10^{-8}$ & $8.9 \times 10^{-8}$ & $8.8 \times 10^{-8}$ & $9.1 \times 10^{-8}$ \\
\hline $\operatorname{Pr}$ & $1.2 \times 10^{1}$ & $1.16 \times 10^{2}$ & $1.16 \times 10^{3}$ & $1.07 \times 10^{5}$ & $8.3 \times 10^{1}$ \\
\hline $\mathrm{d} \sigma / \mathrm{d} T\left(\mathrm{~N} \mathrm{~m}^{-1} \mathrm{~K}^{-1}\right)$ & $-7.63 \times 10^{-5}$ & $-8.28 \times 10^{-5}$ & $-9.5 \times 10^{-5}$ & $-3.77 \times 10^{-5}$ & - \\
\hline$\Delta H \mu / \bar{R}(\mathrm{~K})$ & 1093.5 & 1660.4 & 2138.9 & 5582.9 & - \\
\hline$\mu_{0}(\mathrm{~Pa} \mathrm{~s})$ & $8.7 \times 10^{-4}$ & $9.6 \times 10^{-3}$ & $9.8 \times 10^{-2}$ & 6.74 & \\
\hline$\Delta H_{\mathrm{v}}\left(\mathrm{J} \mathrm{mol}^{-1}\right)$ & 12896 & 60837 & 66531 & 82903 & 108653 \\
\hline$M_{\mathrm{w}}\left(\mathrm{kg} \mathrm{kmol}^{-1}\right)$ & 237 & 1250 & 5970 & 62700 & 950 \\
\hline$T_{\text {sat }}(\mathrm{K})$ & 284 & 290 & 295 & 573 & 510 \\
\hline
\end{tabular}

Data include $\left(\mu_{\mathrm{f}}\right)$ viscosity; $\left(\rho_{\mathrm{f}}\right)$ density; $\left(\sigma_{\mathrm{f}}\right)$ surface tension; $\left(k_{\mathrm{f}}\right)$ thermal conductivity; $\left(\alpha_{\mathrm{f}}\right)$ thermal diffusivity; (Pr) Prandtl number; $(\mathrm{d} \sigma / \mathrm{d} T)$ variation of surface tension with temperature; $\left(\Delta H_{\mu} / \bar{R}\right)$ and $\left(\mu_{0}\right)$ constants for variation of viscosity with temperature; $\left(\Delta H_{\mathrm{v}}\right)$ heat of evaporation; $\left(M_{\mathrm{w}}\right)$ molecular weight; and $\left(T_{\text {sat }}\right)$ saturation temperature. 


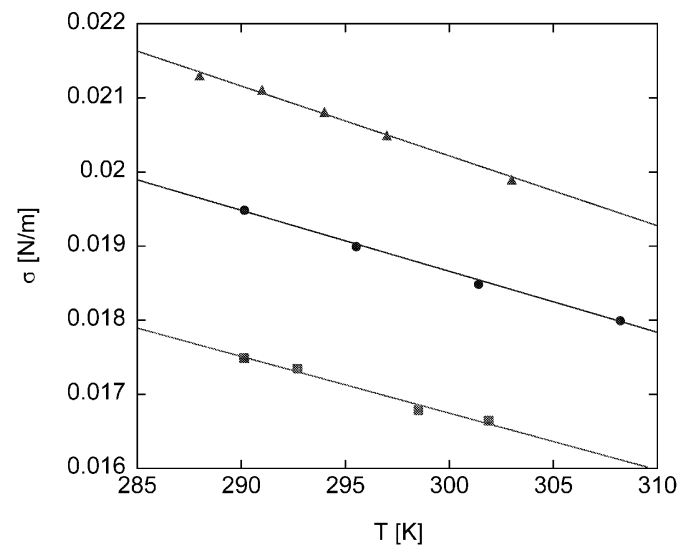

(a)

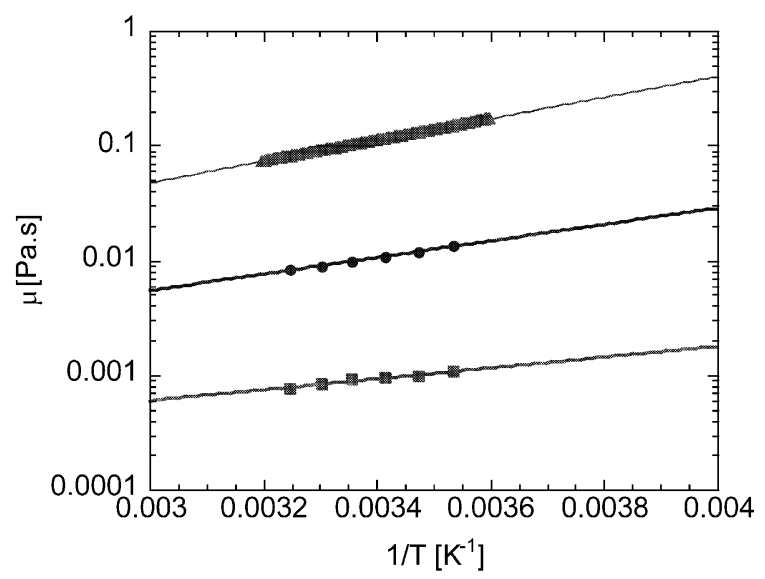

(b)

Fig. 1. Dependence of (a) surface tension and (b) viscosity of silicone oil test fluids on temperature. Data include (ם) L; ( $\mathrm{M} ;(\boldsymbol{\Delta}) \mathrm{H}$.

reflected light passing through the pinhole and received by the light-receiving photodetector is maximized. When the surface is not in focus, the spot size reflected onto the detector plane, containing the pinhole, becomes larger and the transmitted intensity is less. A feedback loop is then used to maintain the maximum intensity.

Calibration tests using micromachined silicon targets show that the resolution of this method is approximately $0.5 \mu \mathrm{m}$ for measurements normal to the surface and the diameter of the spot at best focus is approximately $7 \mu \mathrm{m}$. The response time of the feedback system is $2.2 \mathrm{~ms}$. The power output of the laser is $20 \mu \mathrm{W}$. Our calculation shows that the mass flux caused by incident laser heating is approximately $3.6 \times 10^{-10} \mathrm{~kg} \mathrm{~s}^{-1}$ which is negligible compared with the evaporative mass flux.

Drop spreading experiments were performed using a syringe pump to deliver a liquid drop of precise volume, $\Omega$, on the clean solid substrate. The needle tip was positioned with manipulators to be just above (but not contacting) the substrate so that the initial momentum of impact of the droplet is negligible. When the spherical drop made contact with the solid it began to wet the surface. During this process, the confocal measurement system was focused on the free surface of the spreading drop at the desired point (e.g. close to the center of drop), as shown in Fig. 2. The analog output of the measuring instrument is digitized using an $\mathrm{A} / \mathrm{D}$ card controlled by the LabView program and corresponds to the evolution of the local thickness of the drop $h(X, t)$ as a function of time. The substrate can be translated laterally either in discrete increments or at constant velocity using an automated XY stage (Compumotor, ZETA4 drive with encoder) with a positioning resolution of $\pm 0.5 \mu \mathrm{m}$.

Since the thermal diffusivity of the substrate (with thickness of $1.5 \mathrm{~mm}$ ) is a few orders of

Table 2

Thermal and physical properties of substrate materials

\begin{tabular}{|c|c|c|c|c|}
\hline Substrate material & 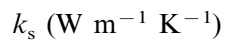 & $\varepsilon_{\mathrm{s}}(\mu \mathrm{m})$ & $C_{\mathrm{p}}\left(\mathrm{J} \mathrm{kg}^{-1} \mathrm{~K}^{-1}\right)$ & $\alpha_{\mathrm{s}}\left(\mathrm{m}^{2} \mathrm{~s}^{-1}\right)$ \\
\hline Copper & 399 & 10,1 & 383 & $1.16 \times 10^{-4}$ \\
\hline Silicon & 153 & 0.01 & 703 & $9.34 \times 10^{-5}$ \\
\hline Brass & 111 & 1 & 385 & $3.41 \times 10^{-5}$ \\
\hline Glass & 0.81 & 0.01 & 800 & $3.40 \times 10^{-7}$ \\
\hline
\end{tabular}

Data include $\left(k_{\mathrm{s}}\right)$ thermal conductivity; $\left(\varepsilon_{\mathrm{s}}\right)$ average roughness; $\left(C_{\mathrm{p}}\right)$ specific heat; and $\left(\alpha_{\mathrm{s}}\right)$ thermal diffusivity. Copper substrates with two surface roughness were used in the experiments. 


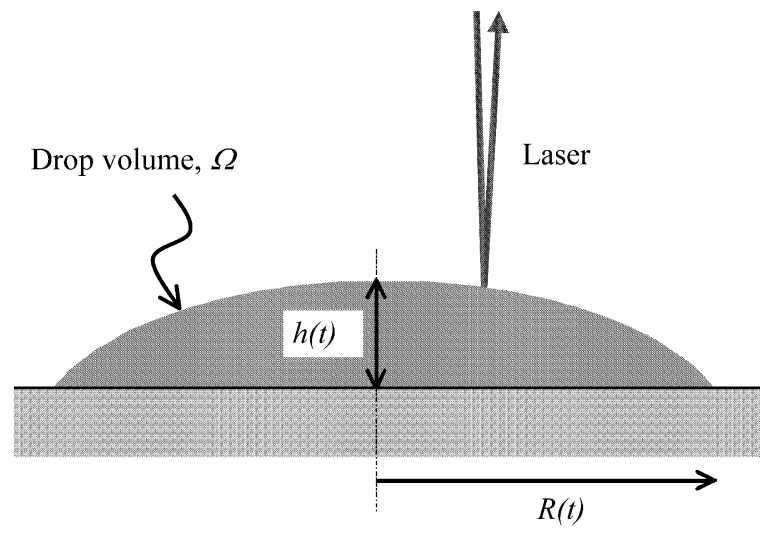

Fig. 2. Schematic diagram of spreading drop and definitions of important length scales.

magnitude larger than that of the liquids, it is reasonable to assume that the temperature of the substrate is equal to the ambient room temperature $(300 \mathrm{~K})$ and remains constant during the course of the experiment. The convection of heat to the air from the free surface of the spreading liquid is negligible compared with the heat of evaporation for the evaporating drops.

\section{Steady spreading}

\subsection{Spreading regimes}

When a viscous drop of wetting liquid is placed on a solid surface, the evolution of the droplet shape depends on its physical properties and its initial volume. The Ohnesorge number, $\mathrm{Oh}=\mu$ / $(\rho R \sigma)^{1 / 2}$, scales the forces that resist the spreading. For $\mathrm{Oh} \gg 1$ the spreading is resisted primarily by viscous stresses in the fluid, whereas at low $\mathrm{Oh} \ll 1$ the spreading is retarded principally by fluid inertial effects. The dominant force driving the spreading depends on the relative size of the drop compared with the capillary length $\ell_{\text {cap }}=(\sigma)$ $\rho g)^{1 / 2}$. In Fig. 3 we show three regimes for the evolution of spreading viscous droplets. If the characteristic length scale for a drop of volume $\Omega$

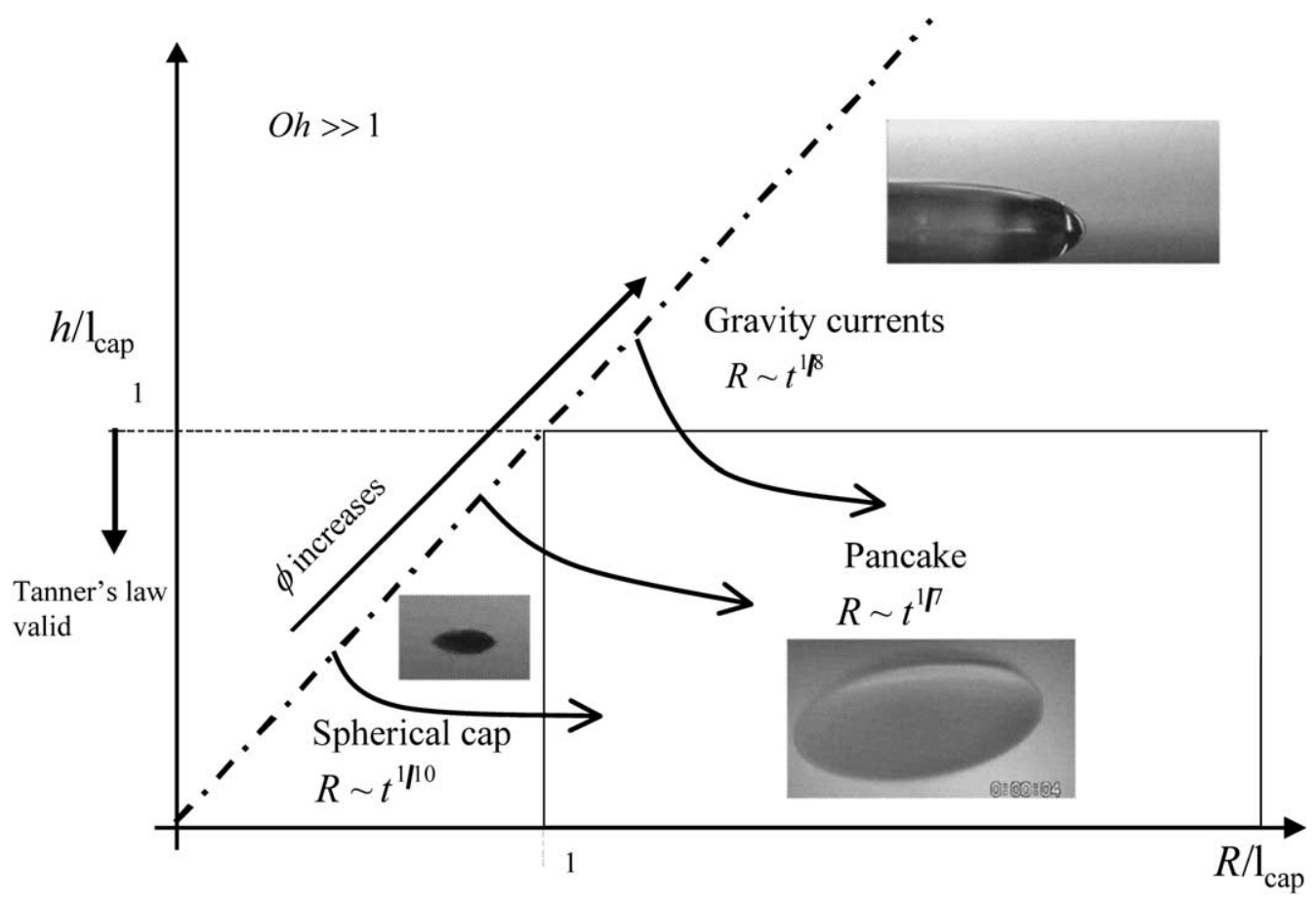

Fig. 3. Three characteristic regimes of evolution for spreading drops of a viscous fluid $\left(\mathrm{Oh}=\mu /(\rho R \sigma)^{\frac{1}{2}} \gg 1\right)$. The dynamics in each regime depend on the relative magnitudes of the height, $h(t)$, the radius, $R(t)$, and the capillary length, $\ell_{\text {cap }}=\sqrt{\sigma / \rho g}$. 
is taken as $(\Omega / \pi)^{1 / 3}$. then an appropriate measure of the dimensionless drop size is $\phi=(\Omega / \pi)^{1 / 3} / \ell_{\text {cap }}$. For small viscous drops $(\phi<1)$, the spreading is driven by capillary force imbalance at the contact line, and resisted by viscosity (for Oh $\gg 1$ ). However, for larger viscous drops $(\phi>1)$, spreading is initially driven by the gravitational body force instead of capillary forces. For the conditions considered in the present study, the impact velocity has negligible effects.

The three regimes shown in Fig. 3 for a viscous drop with $\mathrm{Oh} \gg 1$ depend on the relative magnitudes of the height, $h(t)$, the radius, $R(t)$, compared with the capillary length, $\ell_{\text {cap }}=(\sigma / \rho g)^{1 / 2}$. Small drops with $h<R<\ell_{\text {cap }}$ assume the shape of a spherical cap. In this regime, capillarity acts as the driving force and viscous flow throughout the spherical cap provides the resistance. Scaling arguments yield a power law for variation of the drop radius as a function of time of the form $R(t) \sim t^{1 / 10}$ (Tanner [3]). For larger initial volumes $(\phi>1)$ the drop initially spreads as a 'gravity current' with $\ell_{\text {cap }}<h<R$. Evolution of the drop radius as a function of time can be found by balancing the gravitational body force with viscous stresses and yields $R(t) \sim t^{1 / 8}$ (see, for example, Huppert [6]).

After a long time, the thickness of the spreading drop becomes very small and both of the regimes described above evolve towards a 'pancake regime' with $h<\ell_{\text {cap }}<R$. In this regime, capillarity and viscous stresses are important in an annular region close to the contact line (of lateral extent $\Delta r \sim \ell_{\text {cap }}$ from the contact line). Gravitational forces once again become important in the rest of the drop but only serve to act as a hydrostatic force, leading to a 'pancake-like' central region of constant thickness. In this regime the power law spreading is of the form $R(t) \sim t^{1 / 7}$ (Cazabat and Cohen Stuart [7]).

To test the capabilities of the confocal measurement system, a viscous drop of silicone oil (fluid $\mathrm{H}, \mathrm{Oh}=0.98, \phi=0.86$ ) was placed on a silicon surface and by using the XY translation stage, the free surface profile of the spreading drop was scanned repeatedly as it spread. The scanning speed of the stage is $1 \mathrm{~mm} \mathrm{~s}^{-1}$ and is fast enough that the profile can be assumed to be quasi-steady

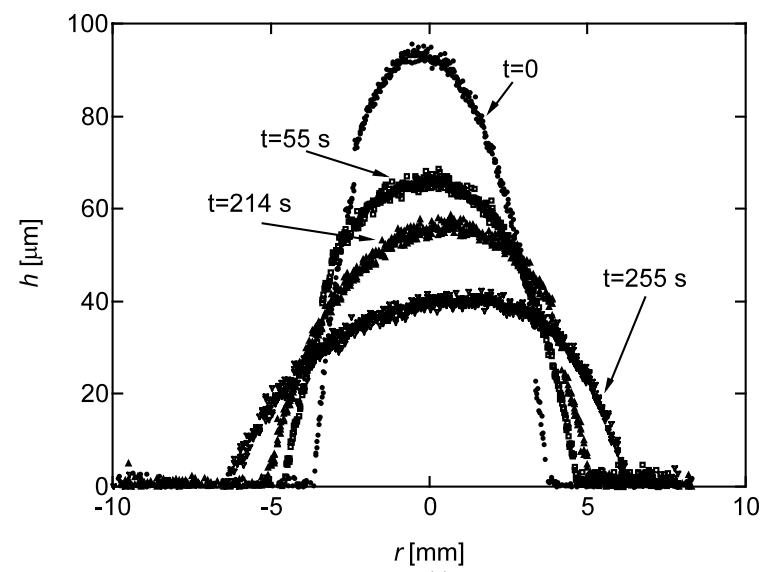

(a)

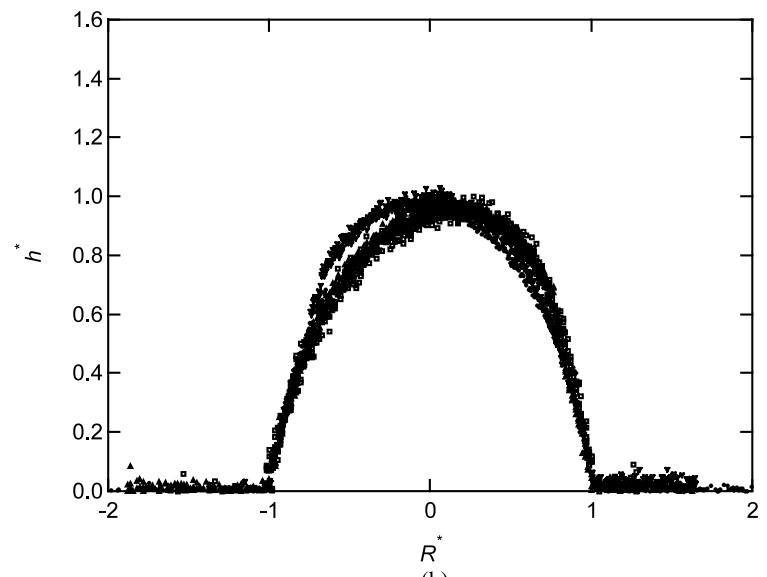

(b)

Fig. 4. (a) Evolution of the free surface, $h(r, t)$, of a spreading droplet of silicone oil $(\mathrm{Oh}=0.98, \phi=0.86)$ on a silicon substrate. Measurement is done by scanning the free surface at a scanning speed of $U=1 \mathrm{~mm} \mathrm{~s}^{-1}$. (b) Normalized height, $h^{*}=h(r, t) / h_{\max }(t)$, vs. normalized lateral position, $R^{*}=r /$ $R(t)$, for the same data set.

over each scan. The results are shown in Fig. 4(a) for $t=0,55,214,255 \mathrm{~s}$ after dispensing the drop. On the abscissa, we show the measured distance from the center of drop, $r$, in $\mathrm{mm}$ and on the ordinate axis we plot the local thickness of the drop, $h$, in $\mu \mathrm{m}$. By normalizing the height profiles by the maximum thickness at each instant, $h^{*}=$ $h(r, t) / h_{\max }(t)$, and the radius by the maximum radius, $R^{*}=r / R(t)$, all of the results of Fig. 4(a) collapse to form a single self-similar profile as shown in Fig. 4(b). Given this self-similarity in the spatial profile of the spreading fluid droplet, it is 
sufficient to monitor the temporal dynamics of the process by using the confocal system to measure the evolution in the height at a single fixed spatial position, and we employ this approach in the majority of the results we present below.

\subsection{Power law results for steady spreading}

The different powerlaw regimes of spreading for viscous drops have been outlined above and we have also validated these experimentally. Many of the previous experiments were done by observing the radius of the drop as it spreads on the substrate by using imaging systems such as CCD cameras. Our measurement system enables us to measure the local thickness of the drop and derive the associated power-law from this data. The power-law coefficients for the radial extent and local thickness are constrained by the selfsimilarity of the shape and conservation of mass so that $\pi R^{2}(t) h(t) \sim \Omega$ at all times.

There are analogous power-law relations for the inertial-capillary spreading of less-viscous drops $(\mathrm{Oh} \ll 1)$. For fluid droplets of low viscosity, the resistance to spreading from viscous stresses is negligible and inertial resistance balances the driving force of capillarity. Viscous drops $(\mathrm{Oh} \gg 1)$ spread much more slowly compared with relatively inviscid drops as expected because the large viscous forces in the thin film retard the spreading. At long times, the volume of the drop $(\phi)$ is not a factor since all spreading drops will eventually move to the pancake regime regardless of initial size (cf. Fig. 3). Table 3(a) shows the approximate form of the similarity solution for spreading at low and high Ohnesorge numbers in the pancake regime $h<\ell_{\text {cap }}<R$.

In Fig. 5 we show experimental spreading results for three different silicone oils with small, medium, and large Ohnesorge numbers. The vertical axis of the plot is the local thickness of the film, $h(t)$, and the horizontal axis of this plot is the elapsed time. For silicone oil droplets spreading at very large Ohnesorge number (fluid $\mathrm{V}$, $\mathrm{Oh}=92, \phi=1.8)$, the analytical power-law result in Table 3 is of the form $h / \ell_{\text {cap }}=A\left(t /\left(\mu \ell_{\text {cap }} /\right.\right.$ $\sigma))^{-2 / 7}$. For the silicone oil with lowest Ohnesorge number $(\mathrm{L}, \mathrm{Oh}=0.003, \phi=1.2)$, the analytical form of the power-law in the pancake regime is $h / \ell_{\text {cap }}=A\left(t /\left(\rho R^{3} / \sigma^{1 / 2}\right)\right)^{-4 / 3}$ as shown in Table 3(a). Linear regression of a power-law to the experimental results shows that these forms of spreading equations accurately describe the experimental observations. The power-law coefficients and the front factors obtained from experiments and from theory are shown in Table 3(b). For fluids with intermediate Ohnesorge numbers close to one (e.g. fluid $\mathrm{H}, \mathrm{Oh}=0.98, \phi=1.4$ ), there is no analytical power-law form available for the evolution of a spreading drop since viscous, capillary and inertial effects are all equally important. However, the power-law coefficient may be expected to lie between the two extreme cases described above. The values obtained from the

Table 3

(a) Theoretical power-laws for viscous-capillary and inertia-capillary spreading of droplet on smooth solid surface. (b) Comparison between the analytical spreading result and the experimental spreading result in the pancake regime

\begin{tabular}{|c|c|c|c|}
\hline (a) Driving force & Resisting force & $\mathrm{Oh}$ & Scaling form \\
\hline Capillary & Viscous & $\mathrm{Oh} \gg 1$ & $h / \ell_{\mathrm{cap}} \cong\left((\Omega / \pi)^{1 / 3} / \ell_{\mathrm{cap}}\right)^{9 / 7}\left(t /\left(\mu \ell_{\mathrm{cap}} / \sigma\right)\right)^{-2 / 7}$ \\
\hline Capillary & Inertia & $\mathrm{Oh} \ll 1$ & $h / \ell_{c a p} \cong\left((\Omega / \pi)^{1 / 3} / \ell_{c a p}\right)^{3}\left(t /{\sqrt{\rho \ell_{\text {cap }}^{3} / \sigma^{-4 / 3}}}^{-4}\right.$ \\
\hline (b) Liquid & $\mathrm{Oh}$ & Analytical result & Experimental result \\
\hline $\mathrm{L}$ & 0.003 & $A=2.32, B=-1.33$ & $A=2.97, B=-1.28$ \\
\hline $\mathrm{H}$ & 0.98 & NA & $A=2.00, B=-0.36$ \\
\hline $\mathrm{V}$ & 92 & $A=0.71, B=-0.28$ & $A=0.56, B=-0.28$ \\
\hline
\end{tabular}

Power-law has the form of $h / \ell_{\text {cap }}=\mathrm{At}{ }^{* \mathrm{~B}}$. Data include V $(\mathrm{Oh}=92, \phi=1.8) ; \mathrm{H}(\mathrm{Oh}=0.98, \phi=1.4) ; \mathrm{L}(\mathrm{Oh}=0.003, \phi=1.2)$. 


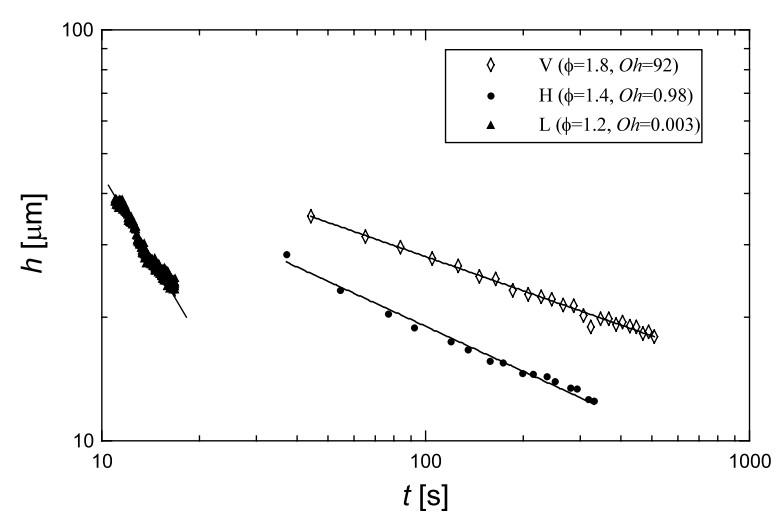

Fig. 5. Comparison of spreading experiments with the scaling analysis. Data include $(\diamond) \mathrm{V}(\mathrm{Oh}=92, \phi=1.8) ;$ (口) $\mathrm{H}$ $(\mathrm{Oh}=0.98, \phi=1.4) ;(\boldsymbol{\Delta}) \mathrm{L}(\mathrm{Oh}=0.003, \phi=1.2)$.

experiment confirm that the power-law coefficient lies between the limiting values of inertia-capillary and viscous-capillary spreading.

\section{Marangoni instability of spreading volatile liquids}

During our experiments on spreading drops it was noticed that if a drop of volatile wetting liquid was deposited on a surface and allowed to spread, then, after a few seconds, periodic fluctuations at the free surface of the liquid film were detected by the confocal measuring system. A series of experiments described below show that these instabilities arise as a result of the volatility of the liquid and the non-uniform evaporation rate across the drop.

The latent heat of spreading and the latent heat of evaporation can both be important in such problems. However, our calculation shows the latent heat of spreading is negligible compared with the latent heat of evaporation. For example, using material properties from Table 1 the latent heat of spreading, $\Delta H=-\sigma+T \partial \sigma / \partial T$, can be calculated as $-40 \mathrm{~mJ} \mathrm{~m} \mathrm{~m}^{-2}$. This means to change the temperature in drop of silicone oil by $1 \mathrm{~K}$, the area of spreading has to be $2 \mathrm{~m}^{2}$ (Hiemenz and Rajagopalan [24]).

A detailed thermal analysis (Oron et al. [5]) shows that the important dimensionless parameter in this type of evaporatively-driven instabilities is the dimensionless interfacial thermal resistance,
$\mathfrak{R}=k_{\mathrm{f}} /\left(\mathscr{H}_{\mathrm{m}} h_{0} \Delta H_{\mathrm{v}}\right)$. Here $k_{\mathrm{f}}$ is the thermal conductivity of the liquid, $h_{0}$ is the local film thickness, $\Delta H_{\mathrm{v}}$ is the latent heat of evaporation in $\mathrm{J} \mathrm{kg}^{-1}$, and $\mathscr{H}_{\mathrm{m}}$ is the coupled heat/mass transfer coefficient in $\mathrm{kg}$ $\mathrm{m}^{-2} \mathrm{~s}^{-1} \mathrm{~K}^{-1}$. A detailed kinetic theory shows that this parameter is given by $\mathscr{H}_{\mathrm{m}}=\hat{\alpha} \rho_{\mathrm{g}} \Delta H_{\mathrm{v}}\left(M_{\mathrm{w}} /\right.$ $2 \pi \bar{R})^{1 / 2} / T_{\text {sat }}^{3 / 3}$ where $\rho_{\mathrm{g}}$ is the density of the vapor in $\mathrm{kg} \mathrm{m}^{-3}, \hat{\alpha}$ is the accommodation coefficient (typically $\approx 1), T_{\text {sat }}$ is the saturation temperature of the vapor at a given pressure, $\bar{R}$ is the universal gas constant, and $M_{\mathrm{w}}$ is the molecular weight of the vapor (for further details see Palmer [25]).

This dimensionless parameter $\mathfrak{R}$ quantifies the relative magnitude of the evaporative resistance to heat transfer at the interface compared with the conductive resistance of the liquid film itself. It is analogous to the inverse of the Biot number encountered in convection-diffusion heat transfer problems, $\mathrm{Bi}=h_{\text {conv }} /\left(k_{\mathrm{s}} / h_{0}\right)$, in which $h_{\text {conv }}$ is the heat transfer coefficient for convection into a liquid and $k_{\mathrm{s}} / h_{0}$ is the heat transfer coefficient for thermal diffusion through the solid boundary.

A linear stability analysis (Kavehpour and McKinley [26]) shows that for $\mathfrak{R}_{\text {critical }} \leq \mathfrak{R}<\infty$ disturbances on the spreading film are amplified and for $\mathfrak{R}<\mathfrak{R}_{\text {critical }}$ the liquid microdroplets spread without showing this type of evaporative instability. The limit $\mathfrak{R}=0$ is special and corresponds to the case of a perfectly thermally-insulating film or a non-volatile spreading fluid with zero mass flux from the surface.

There are several thermophysical parameters of both the spreading fluid and the underlying substrate that affect the observed instability phenomenon. In the following sections, we explore each of these factors.

\subsection{Effect of liquid viscosity}

In Fig. 6 we show the evolution in the height of the spreading droplets close to onset of instability for (a) $\mathrm{L}(\mathrm{Oh}=0.003, \phi=1.39)$, (b) $\mathrm{M}(\mathrm{Oh}=0.02$, $\phi=1.35)$, and (c) $\mathrm{H}(\mathrm{Oh}=0.23, \phi=1.33)$. In each figure the ordinate axis is the local thickness of the drop in micrometers and the abscissa represents elapsed time in seconds. The solid lines show the power-law relation expected for the spreading in the absence of Marangoni instability. 
For the liquid with the lowest viscosity (Fig. 6(a)) the instability starts at a film thickness of about $30 \mu \mathrm{m}$. The insets to the figure show details of the periodic fluctuations in the free surface height of the drop. It can be seen that as the drop spreads and the film thickness decreases the relative amplitude of the oscillations increases and the frequency decreases. In Fig. 6(b) we show the onset of instability in the oil of intermediate viscosity (M). It can be seen that the instability threshold is achieved at a lower critical thickness than observed in the previous case. Also the amplitude of oscillations is smaller than observed in the low Oh case. The inset to Fig. 6(b) shows the

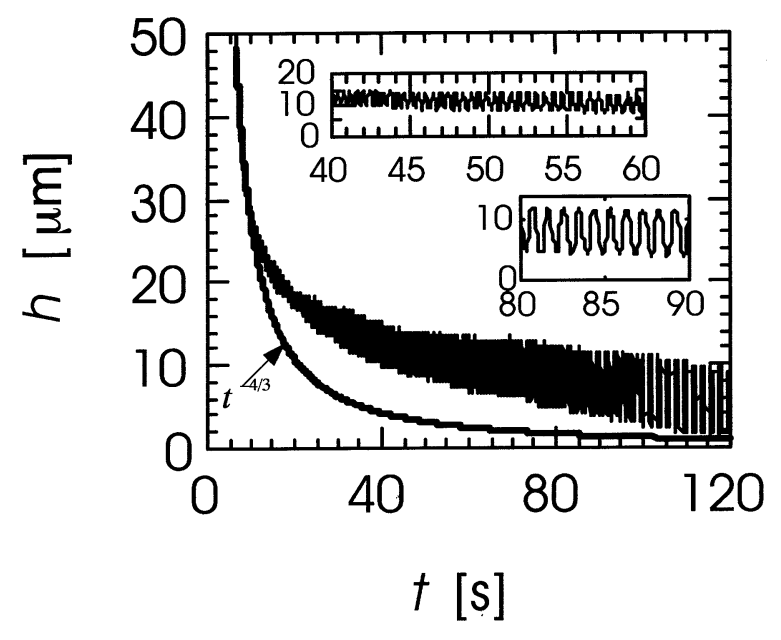

(a)

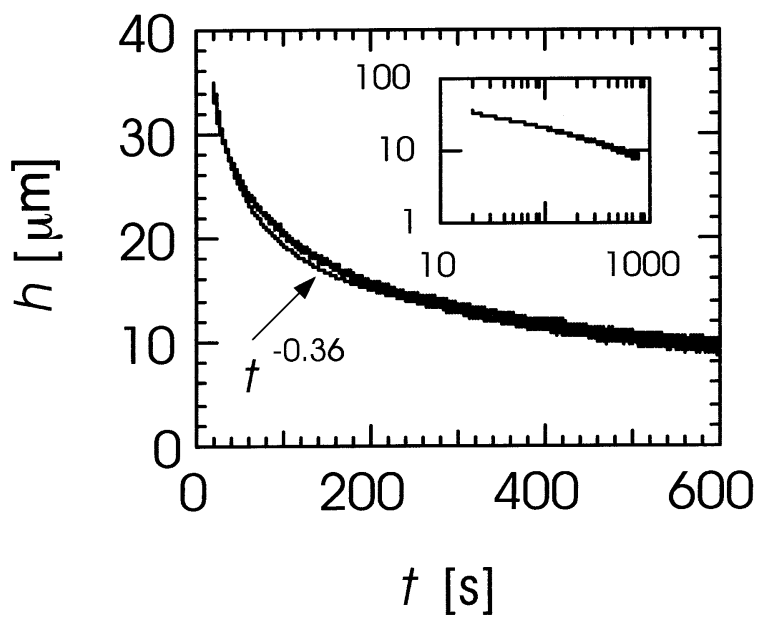

(b)

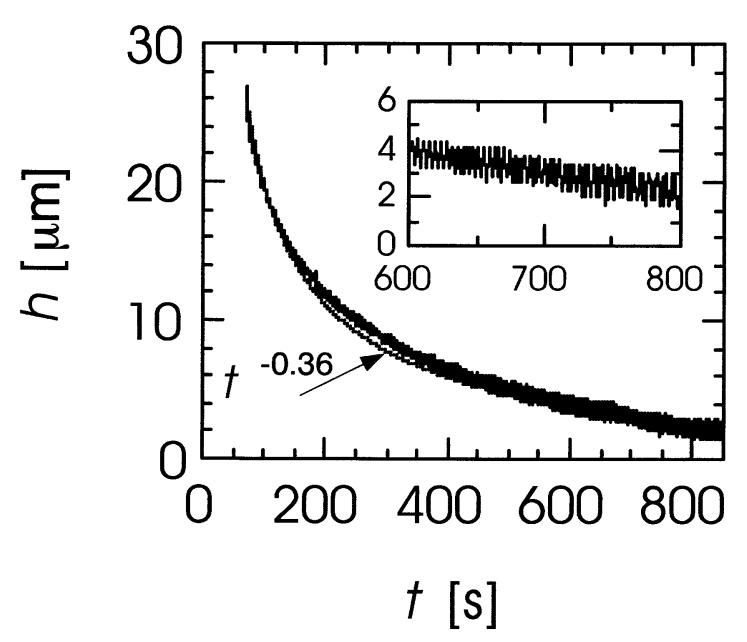

(c)

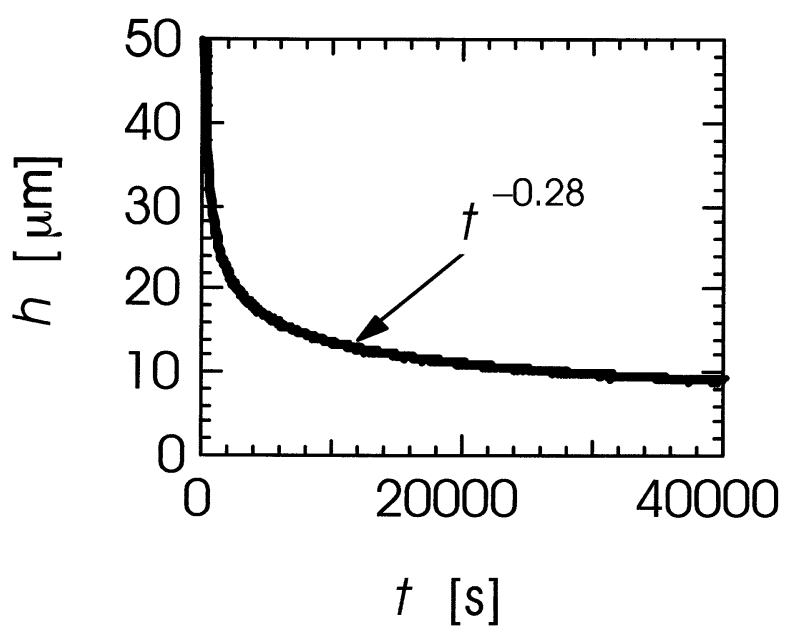

(d)

Fig. 6. Onset of instability for fluids of low, medium and high viscosity (a) $\mathrm{L}(\mathrm{Oh}=0.003, \phi=1.39)$; (b) $\mathrm{M}(\mathrm{Oh}=0.02, \phi=1.35)$; and (c) $\mathrm{H}(\mathrm{Oh}=0.23, \phi=1.33)$. The solid lines show the power law relation expected for the spreading in the absence of instability. (d) Spreading profile for fluid $\mathrm{V}(\mathrm{Oh}=62.9, \phi=1.31)$. No instability can be observed within the resolution of the measuring system. Dotted line is the expected power-law relation expected from the analysis. 
same data plotted on double logarithmic axes. Initially, the film thickness has a powerlaw form as expected. As the disturbance grows there is a small but systematic deviation from the simple powerlaw spreading. In Fig. 6(c) we show the onset of instability in the more viscous fluid $(\mathrm{H})$. The same trend can be seen here as the instability starts at a yet lower film thickness and the amplitude of the oscillations are smaller than those observed at lower Oh. Finally, in Fig. 6(d) we show the spreading profile observed in the most viscous fluid (V). No instability can be observed within the resolution of the measuring system down to the minimum detectable height of approximately $0.5 \mu \mathrm{m}$.

\subsection{Directionality of the traveling waves}

The periodic disturbances shown in Fig. 6(a-c) correspond to surface perturbations propagating past the fixed measuring point of the laser confocal microscope. To investigate the speed and direction of the surface waves generated by this evaporative Marangoni instability, the surface

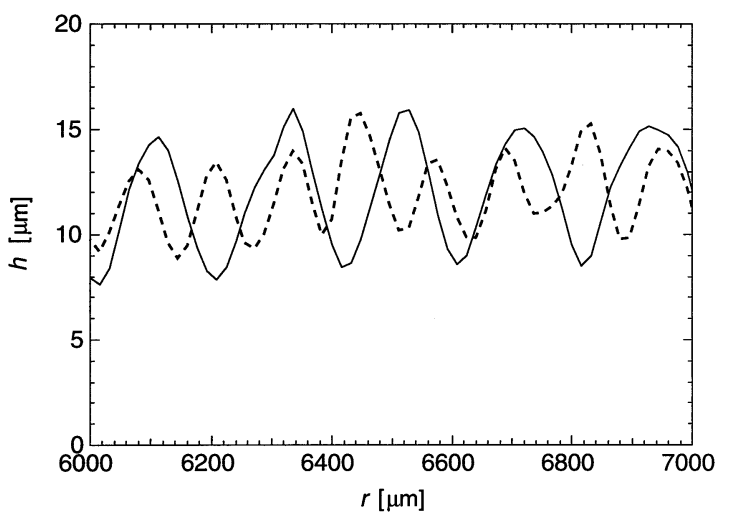

Fig. 7. Scanning a droplet of silicone oil $(\mathrm{L}, \mathrm{Oh}=0.003$, $\phi=1.4)$ spreading on silicon substrate with scanning speed of $320 \mu \mathrm{m} \mathrm{s}^{-1}$ with the direction (-) from contact line toward the center and (_ _ - ) from center toward the contact line.

profile of a spreading droplet of low viscosity silicone oil (L) is measured by scanning the laser probe across the drop at a constant speed of $U=320 \mu \mathrm{m} \mathrm{s}^{-1}$. First the film surface is scanned from the contact line toward the center of the drop and then in the reverse direction from its

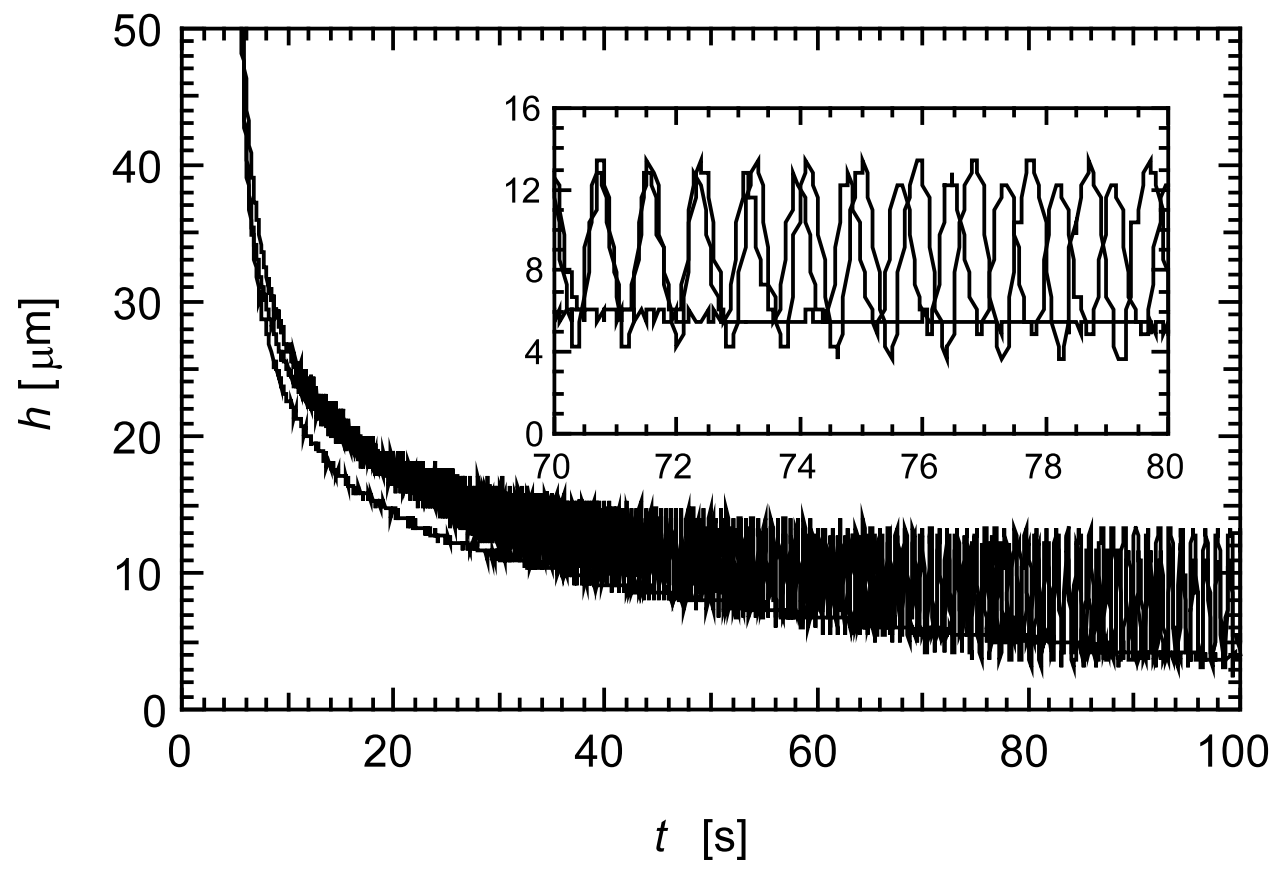

Fig. 8. Effect of thermal conductivity of substrate on the onset of instability. Spreading of silicone oil $(\mathrm{L}, \mathrm{Oh}=0.003, \phi=1.39)$ on (...) glass; (_ _ _ $)$ brass; and $\left({ }_{-}{ }_{-}\right)$silicon substrates. 
center toward the contact line. The scanning results are shown in Fig. 7. The solid line shows the wave profile when the scanning direction is from the leading edge towards the center and the dashed line represents the wave pattern when the scanning direction is from the center to the contact line. It can be seen in this figure that the waves have a higher apparent spacial frequency when the scanning direction is from the center outwards towards the contact line. This Doppler shifting in the disturbance frequency indicates that the surface waves resulting from the evaporatively-driven Marangoni instability propagate radially-inwards from the contact line to the center of the drop.

\subsection{Effect of substrate thermal conductivity}

Several materials have been used as substrates in our experiments and their thermophysical properties are found to have a pronounced effect on this new type of Marangoni instability. In Fig. 8 we show the effect of the thermal conductivity of the substrate on the stability characteristics. Three different smooth substrates were used to observe the spreading of the lowest viscosity silicone fluid (L). The thermal diffusivities of the selected materials are: glass $\left(s=3.40 \times 10^{-7} \mathrm{~m}^{2} \mathrm{~s}^{-1}\right)$, brass $\left(s=3.41 \times 10^{-5} \mathrm{~m}^{2} \mathrm{~s}^{-1}\right)$, and silicon $(s=9.34 \times$ $\left.10^{-5} \mathrm{~m}^{2} \mathrm{~s}^{-1}\right)$. The thermal diffusivity of silicon and brass are close to each other and both are good thermal conductors. The instabilities that develop during the spreading of a volatile liquid on these surfaces are very similar, the amplitudes of oscillation are almost identical and the critical film thickness for onset of instability are very close to each other. However, although the silicon fluid readily wets the smooth glass surface, the substrate material is a thermal insulator with a much smaller thermal diffusivity. No surface oscillations are observed during the steady spreading of the drop across the glass surface. It is thus clear that heat transfer from the underlying substrate is very important in driving the onset of Marangoni instability in the fluid film.

\subsection{Effect of surface roughness}

The experiments described above have all been

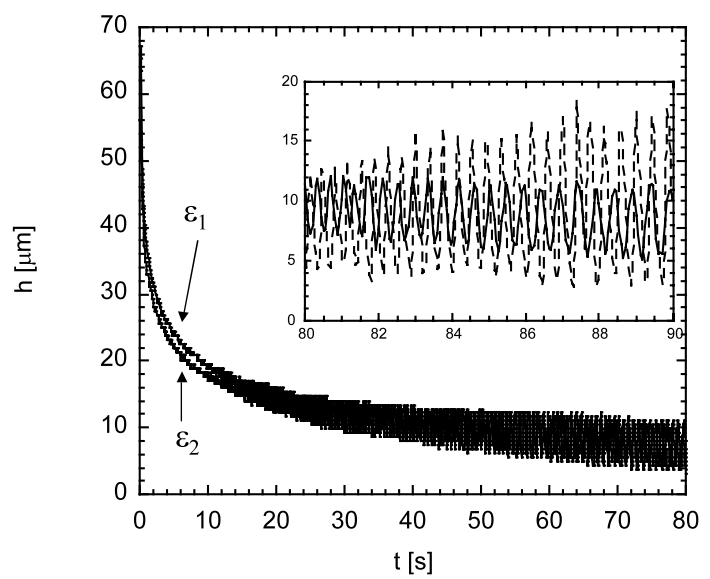

Fig. 9. Effect of substrate roughness on spreading and instability. Silicone oil $(\mathrm{L}, \mathrm{Oh}=0.003, \phi=1.39)$ drop spreading on copper substrates of characteristic roughness $(-) \varepsilon_{1}=1.13 \times$ $10^{-6} \mathrm{~m}$, and $(--\longrightarrow) \varepsilon_{2}=1.83 \times 10^{-5} \mathrm{~m}$.

carried out on relatively smooth surfaces. It is already known that changes in surface roughness significantly affect the steady spreading of viscous fluids (Cazabat and Stuart [7]). The rate of surface area generation will affect the coupled heat and mass transfer rate arising from evaporation of the fluid. Furthermore, our observations above show that the onset of instability occurs at very small film thickness; the microscopic surface roughness of the substrate may thus also be expected to affect the onset of instability in a volatile liquid. In order to investigate this, we used two different thermallyconductive copper surfaces with controlled degrees of surface roughness and random orientation. Measurements using a surface profilometer (DEKTAK3) gave average roughness values of $\varepsilon_{1}=1.13 \times 10^{-6}$ and $\varepsilon_{2}=1.83 \times 10^{-5}$. These surfaces were then used as substrates to investigate spreading of a liquid micro-droplet of the least viscous and most unstable fluid $(\mathrm{L} ; \mathrm{Oh}=0.003$, $\phi=1.39)$.

In Fig. 9 we demonstrate the effect of surface roughness on the thermocapillary instability of volatile liquids during the spreading process. Prior to onset of instability, the rate of steady spreading is observed to be slower for the surface with smaller surface roughness than for the substrate with higher surface roughness. The critical film thickness 
at onset of instability appears to be approximately the same for both cases $\left(h_{\text {crit }} \approx 20 \mu \mathrm{m}\right)$ but the amplitude of oscillations on the rough surface are substantially larger. Details of the surface perturbations are shown in the inset to Fig. 9. Fourier analysis of such local signal segments shows that the waveform of the oscillations for the lower surface roughness case are essentially sinusoidal in nature whereas the disturbances propagating over the substrate with high surface roughness develop strongly non-linear characteristics when the film becomes very thin.

\subsection{Effect of liquid volatility}

We have argued above (Section 4.1) that this instability is a Marangoni instability driven not by imposed temperature gradients but by gradients arising naturally as a result of evaporation. The latent heat of evaporation $\left(\Delta H_{\mathrm{v}}\right)$ of the spreading liquid should thus play a central role in the critical onset conditions. To investigate this, we utilized a special grade of silicone oil (GELEST, DMS-T07R) with comparable properties to fluids $\mathrm{L}$ and $\mathrm{M}$ but with a much lower volatility $\left(\Delta H_{\mathrm{v}}^{(\mathrm{DMS}-07 \mathrm{R})}=108.7 \mathrm{~kJ} \mathrm{~mol}^{-1}\right.$, cf. For fluid L, $\left.\Delta H_{\mathrm{v}}^{(\mathrm{L})}=12.9 \mathrm{~kJ} \mathrm{~mol}^{-1}\right)$.

It can be seen from Fig. 10 that the spreading characteristics of the volatile and non-volatile sili-

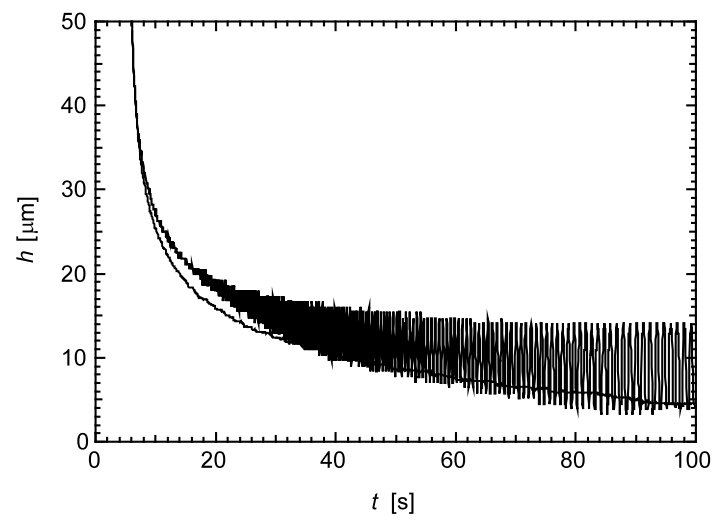

Fig. 10. Effect of volatility of liquid on the onset of instability. Spreading of volatile silicone oil $(-, \mathrm{L}, \mathrm{Oh}=0.003, \phi=1.39$, $\left.\mathfrak{R}_{\mathrm{Vol}}=525 \times 10^{-5} / h_{0}\right)$ and non-volatile silicone oil $(---$, $\left.\mathrm{NV}, \mathrm{Oh}=0.02, \phi=1.3, \mathfrak{R}_{\mathrm{NV}}=8.20 \times 10^{-7} / h_{0}\right)$ on silicon substrate. No instability was observed for non-volatile silicone oil. cone oils on a smooth silicon substrate are dramatically different. At short times, both droplets exhibit the same rapid initial decrease in film thickness. For both fluid droplets the spreading parameters correspond to $\phi>1$ and $\mathrm{Oh} \ll 1$, so that spreading is controlled by a balance of gravitational and inertial forces which are identical for both droplets. As the film spreads out and the surface area increases, evaporative heat and mass transfer losses become increasingly important. The evaporatively-driven Marangoni instability is clearly present for the volatile liquid (L) whereas the non-volatile silicone oil continues to spread steadily on the silicon substrate without indications of surface disturbances.

The parameter controlling onset of instability is the dimensionless interfacial thermal resistance, $\mathfrak{R}=k_{\mathrm{f}} / \mathscr{H}_{\mathrm{m}} h_{0} \Delta H_{\mathrm{v}}$. This parameter can be evaluated using the tabulated values of the thermal conductivity, latent heat of evaporation and the known molecular properties of the silicone oil which control the heat/mass transfer coefficient $\mathscr{H}_{\mathrm{m}}$ (here for simplicity, we assume the accommodation coefficient is $\alpha=1$ ). For the volatile silicone oil (L), the dimensionless interfacial thermal resistance becomes $\mathfrak{R}_{\mathrm{L}}=5.25 \times 10^{-5} / h_{0}$ (with $h_{0}$ the film thickness in meters) whereas for the nonvolatile silicone oil, the larger latent heat of evaporation leads to a value $\mathfrak{R}_{N V}=8.20 \times 10^{-7} / h_{0}$. As each microdroplet spreads, the local thickness decreases, and consequently the interfacial resistance $\mathfrak{R}$ increases. For the volatile silicone oil, $\mathfrak{R}_{\mathrm{L}}$ is two order of magnitudes larger than $\mathfrak{R}_{\mathrm{NV}}$ and thus attains the critical value $\mathfrak{R}_{\text {critical }}$ at a much larger film thickness than the less-volatile fluid.

\section{Discussion and conclusions}

A non-invasive optical technique based on confocal microscopy has been developed and been used to investigate steady spreading and the onset of an evaporatively-driven Marangoni instability of spreading liquid microdroplets. Three characteristic dynamical regimes of steady spreading drops (viscous-capillary, viscous-inertia-capillary, and inertia-capillary) have been studied using this non-invasive optical technique. The dynamics of 
each regime depend on the Ohnesorge number, $\mathrm{Oh}=\mu /(\rho R \sigma)^{\frac{1}{2}}$, and on the relative magnitudes of the droplet height and radius as compared with the capillary length, $\ell_{\text {cap }}=\sqrt{\sigma / \rho g}$. The power-law relationships between the extent of spreading and time extracted from the experiments are in excellent agreement with existing analytical results. This demonstrates that our optical system is an excellent tool for investigating the dynamics of spreading of liquid droplets.

Time-resolved point-wise measurements of the evolution in the free surface of spreading fluid drops have been used to investigate the onset of an evaporatively-driven Marangoni instability. The onset of instability occurs once the height of the liquid film has fallen below a critical value and it results in periodic surface fluctuations which appear to travel radially-inwards towards the droplet center. Experiments with a range of fluids have shown that as the viscosity of the fluid is increased (and the rate of spreading concomitantly decreased) the amplitude of the disturbances is progressively dampened.

The effects of substrate thermal diffusivity and surface roughness on the disturbance characteristics have also been shown in this paper. When droplets spread over substrates with a low thermal diffusivity (such as glass) no instability can be detected whereas for highly thermally-conducting substrates (for example, silicon, copper and brass), ripples were present on the free surface of the spreading droplet. Increasing the surface roughness of the substrate leads to larger amplitude and more non-linear waveforms.

Mathematical modeling of this interfacial instability is complicated due to the coupled mass and heat transfer from the evaporating fluid droplet and the unsteady base flow of the spreading film. A detailed linear stability analysis is currently being performed (Kavehpour and McKinley [26]) using the lubrication framework developed by Ehrhard and Davis [27] and Oron et al. [5]. Physically, the mechanism of instability appears to be the following: evaporation of the volatile material from the fluid film results in a loss of thermal energy from the fluid droplet and a consequent cooling in the droplet. The heat and mass flux are largest near the singular contact line and conse- quently the liquid is coldest here; a surface temperature gradient thus exists along the surface of the thin fluid film which can drive a secondary flow. Under quasi-steady state conditions this evaporative heat and mass flux from the free surface is provided by heat transfer from the underlying substrate and conduction through the insulating liquid film; there is thus also a temperature gradient normal to the fluid layer. The resulting temperature gradients within the drop drive secondary thermocapillary surface flows which can be unstable if the temperature gradient is large enough, although since the gradients exist both parallel and normal to the free surface it is not clear a priori whether to refer to them as Marangoni or hydrothermal instabilities (Schatz and Neitzel [17]). The experimental observations in Fig. 7 showing that the waves propagate towards the central (warmer) region of the droplet suggest that the waves are principally hydrothermal in nature (Smith [28]).

The observed sensitivity of the instability to the thermal properties of the substrate is consistent with the picture above as the magnitude of the heat and mass transfer rate from the surfaceand thus the temperature profile within the filmare constrained by the energy supply from the substrate below. It should be noted that although the free surface instability documented in this paper shares some characteristics with the steady and unsteady secondary flows documented in the coffee drop problem (Deegan et al. [18]) or in tear-ducts of wine (Hosoi and Bush [19]), it is fundamentally different in origin. The interfacial Marangoni stresses that drive the disturbances are not compositional in nature since the spreading silicone oils are single-component liquids. The destabilizing Marangoni stresses arise from the coupled heat and mass transfer resulting from evaporation. Although the thermal dependence of the material properties (especially the surface tension) are essential in this instability, it is not simple to define a critical capillary or Marangoni number since the spreading velocity of the droplet $\dot{R}(t)$ is continuously decreasing with time. Analysis shows that the parameter controlling the temperature profile in the film and thus controlling the onset condition of the thermocapillary insta- 
bility is the dimensionless interfacial thermal resistance, $\mathfrak{R}$. Since the relative magnitude of this interfacial resistance is inversely proportional to the thickness of the liquid droplet, $\mathfrak{R}$ increases as the film spreads and thins. When $\mathfrak{R}$ is larger than a critical value, $\mathfrak{R}_{\text {critical }}$, infinitesimal disturbances on the surface of the spreading drop grow spontaneously. More volatile liquids have larger values of $\mathfrak{R}$ in comparison to non-volatile liquids, due to the smaller latent heat of evaporation. As a consequence, instabilities develop at larger film thickness for more volatile materials. In our experiments, a non-volatile silicone oil did not show instability even at thickness of order $1 \mu \mathrm{m}$ whereas a more volatile silicone oil became unstable at thickness of approximately $20 \mu \mathrm{m}$. From the definitions of $\mathfrak{R}$ and $\mathscr{H}_{\mathrm{m}}$ it can be seen that $\mathfrak{R} \sim\left(\Delta H_{\mathrm{v}}\right)^{-2}$ and thus it would be expected that the non-volatile liquid film will not become unstable until the thickness falls below approximately $0.4 \mu \mathrm{m}$. In the future we hope to be able to probe the dynamic evolution of liquid films to such small submicron scales using the non-perturbative technique of laser-feedback interferometry (Fischer and Ovryn [29]).

\section{Acknowledgements}

The authors would like to thank L. Mahadevan for insightful discussions on the mechanism of instability and S. Chaieb for assistance in some preliminary experimental measurements of spreading liquid films. This research was supported by the National Aeronautics and Space Administration under grant NAG3-2155.

\section{References}

[1] A.W. Adamson, A.P. Gast, Physical Chemistry of Surfaces, sixth ed., Wiley, New York, 1997.
[2] F. Brochard, P.G. de Gennes, J. Physique Lett. 45 (1984) $597-602$.

[3] L.H. Tanner, J. Phys. D: Appl. Phys. 12 (1979) 14731484.

[4] P.G. de Gennes, Rev. Mod. Phys. 57 (1985) 827-863.

[5] A. Oron, S.H. Davis, S.G. Bankoff, Rev. Mod Phys 69 (1997) 931-980.

[6] H.E. Huppert, J. Fluid Mech. 121 (1982) 43-58.

[7] A.M. Cazabat, M.A. Cohen Stuart, J. Phys. Chem. 90 (1986) 5845-5849.

[8] J.A. Fay, in: D.P. Hoult (Ed.), The Spread of Oil Slicks on a Calm Sea, in Oil on the Sea, Plenum, New York, 1969, pp. 53-63.

[9] D.P. Hoult, Ann. Rev. Fluid Mech. 4 (1972) 341-368.

[10] P. Ehrhard, J. Fluid Mech. 257 (1993) 463-483.

[11] C. Redon, F. Brochard-Wyart, F. Rondelez, J. Phys. II France 2 (1992) 1671-1676.

[12] X. Fanton, A.M. Cazabat, Langmuir 14 (1998) 25542561.

[13] L.E. Scriven, C.V. Sternling, Nature 187 (1960) 186-188.

[14] M.K. Smith, S.H. Davis, J. Fluid Mech. 132 (1983) $119-144$.

[15] M.K. Smith, S.H. Davis, J. Fluid Mech. 132 (1983) $145-162$.

[16] S.H. Davis, Ann. Rev. Phys. 19 (1987) 403-435.

[17] M.F. Schatz, G.P. Neitzel, Ann. Rev. Phys. 33 (2001) 93-127.

[18] R.D. Deegan, O. Bakajin, T.F. Dupont, G. Huber, S.R. Nagel, T.A. Witten, Nature 389 (1997) 827-829.

[19] A.E. Hosoi, J.W.M. Bush, J. Fluid Mech. 442 (2001) $217-239$.

[20] H. Hu, R.G. Larson, Fifth Microgravity Fluid Physics and Transport Phenomena Conference, Cleveland, $\mathrm{OH}$, USA, 2000, pp. 109-110.

[21] A.V. Mirzamoghadam, I. Catton, J. Heat Transfer 110 (1988) 208-213.

[22] R. Fondecave, A. Buguin, F. Brochard, CR Acad. Sci. Paris 327 (1999) 407-414.

[23] T.R. Corle, G.S. Kino, Confocal Scanning Optical Microscopy and Related Imaging Systems, Academic Press, San Diego, 1996.

[24] P. Hiemenz, R. Rajagopalan, Principles of Colloid and Surface Chemistry, Marcel Dekker, New York, 1997.

[25] H.J. Palmer, J. Fluid Mech. 75 (1976) 487.

[26] P. Kavehpour, G.H. McKinley, J. Fluid Mech., 2002, in preparation.

[27] P. Ehrhard, S.H. Davis, J. Fluid Mech. 229 (1991) 365388.

[28] M.K. Smith, Phys. Fluids 29 (1986) 3182-3186.

[29] D.G. Fischer, B. Ovryn, Opt. Let. 25 (2000) 478-480. 